\title{
Assignment Problems with fuzzy costs using Ones Assignment Method
}

\author{
S.Vimala ${ }^{1}$, S.Krishna Prabha ${ }^{2}$ \\ ${ }^{1}$ Assistant Professor, Department of Mathematics, Mother Teresa Women's University, Kodaikannal, Tamilnadu \\ ${ }^{2}$ Ph.D Scholar, Mother Teresa Women's University, Kodaikannal. Assistant Professor, Department of \\ Mathematics, PSNA CET, Dindigul, Tamilnadu
}

\begin{abstract}
In this paper we introduce a new approach to solve fuzzy assignment problem namely, ones assignment method. Considering each fuzzy cost as a triangular fuzzy numbers the fuzzy assignment problem has been transformed into crisp values by using linguistic variables and ranking techniques and then it is solved by ones assignment method. The proposed method is a systematic procedure, easy to apply and can be utilized for all types of assignment problem with maximize or minimize objective functions. At the end, this method is illustrated with some numerical example
\end{abstract}

Key words: ranking of fuzzy numbers, triangular fuzzy numbers, Fuzzy Assignment problem, ones method Mathematics Subject Classification: 90C08, 90C10, 03E72,

\section{Introduction}

An assignment problem (AP) is a particular type of transportation problem where $\mathrm{n}$ tasks (jobs) are to be assigned to an equal number of $n$ machines (workers) in one to one basis such that the assignment cost (or profit) is minimum (or maximum).Hence, it can be considered as a balanced transportation problem in which all supplies and demands are equal, and the number of rows and columns in the matrix are identical. Various ranking procedures have been developed since 1975 where the theories of fuzzy sets first introduced by Zadeh [21]. Ranking fuzzy numbers proposed by Jain [13] for decision making in fuzzy situations by representing the ill-defined quantity as a fuzzy set. Since then, various procedures to rank fuzzy quantities are proposed by various researchers. Bortolan and Degani [4] reviewed some of these ranking methods for ranking fuzzy subsets. Chen [6] presented ranking fuzzy numbers with maximizing set and minimizing set. Dubois and Prade presented the mean value of fuzzy number. Lee and Li [16] presented a comparison of fuzzy numbers based on the probability measure of fuzzy events. Chen and Chen [7] derived a new method on ranking generalized trapezoidal fuzzy numbers based on centroid point and standard deviations. Till date, several researchers studied extensively to solve fuzzy assignment problems in various ways.

The triangular fuzzy numbers are defuzzified by using centriod ranking formula .In section 2 some elementary concepts and operations of fuzzy set theory have been reviewed. In section 3, corresponding algorithms have been proposed for Fuzzy assignment problems. In section 4 the proposed method is illustrated by a numerical example

\section{Basic definitions}

2.1Fuzzy set: A fuzzy set is characterized by a membership function mapping element of a domain, space or universe of discourse $X$ to the unit interval $[0,1]$ i.e. $A=\left\{\left(x, \mu_{A}(x) ; x \in X\right\}\right.$, Here $\mu_{A}: X \rightarrow[0,1]$ is a mapping called the degree of membership function of the fuzzy set $A$ and $\mu_{A}(x)$ is called the membership value of $x \varepsilon X$ in the fuzzy set A. These membership grades are often represented by real numbers ranging from $[0,1]$.

\subsection{Fuzzy Number}

A real number $\widetilde{a}$ is a fuzzy subset of the real number $\mathrm{R}$ with membership function $\mu_{\breve{a}}$ satisfying the following conditions,

$\mu_{\breve{a}}$ is continuous from $\mathrm{R}$ to the closed interval $[0,1]$

$\mu_{\breve{a}}$ is strictly increasing and continuous on $\left[\mathrm{a}_{1}, \mathrm{a}_{2}\right]$

$\mu_{\breve{a}}$ is strictly decreasing and continuous on $\left[\mathrm{a}_{3}, \mathrm{a}_{4}\right]$

\subsection{Triangular Fuzzy Number}

The fuzzy number $\widetilde{a}$ is a triangular fuzzy numbers, denoted by $\widetilde{a}=\left(a_{1}, \mathrm{a}_{2}, a_{3}\right)$ its membership function $\mu_{a}$ is given below the figure. 


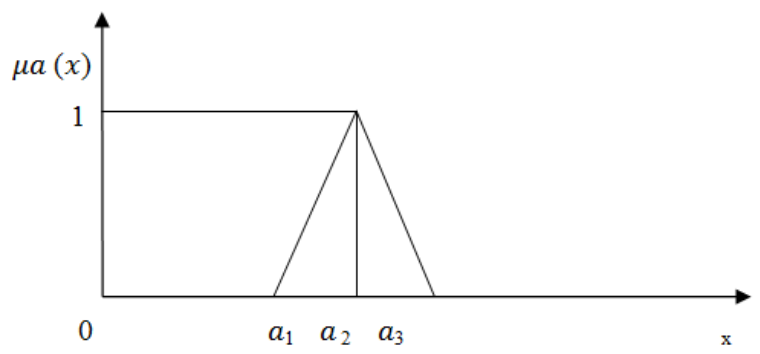

$\mathrm{F}(\mathrm{R})$ represents the set all trapezoidal fuzzy numbers It $\mathrm{R}$ be any ranking function then ,

$R(a)=\left(a_{1}+a_{2}+a_{3}\right) / 3$

Let $a=\left[a_{1}, a_{2}, a_{3},\right]$ and $b=\left[b_{1}, b_{2}, b_{3}\right]$ be two triangular fuzzy numbers then the arithmetic operations on $a$ and $b$ as follows.

\subsection{Properties of Triangular Fuzzy Number}

Addition: $a+b=\left(a_{1}+b_{1}, a_{2}+b_{2},{ }_{3}+b_{3}\right)$

Subtraction: $a-b=\left(a_{1}-b_{1}, \mathrm{a}_{2}-b_{2}, a_{3}-b_{3}\right)$

\section{Multiplication:}
a. $b=\frac{a_{1}}{3}\left(\mathrm{~b}_{1}+b_{2}+b_{3}\right), \frac{a_{2}}{3}\left(\mathrm{~b}_{1}+b_{2}+b_{3}\right), \frac{a_{3}}{3}\left(\mathrm{~b}_{1}+b_{2}+b_{3}\right)$ if $R(a)>0$
$a \cdot b=\frac{a_{3}}{3}\left(\mathrm{~b}_{1}+b_{2}+b_{3}\right), \frac{a_{2}}{3}\left(\mathrm{~b}_{1}+b_{2}+b_{3}\right), \frac{a_{1}}{3}\left(\mathrm{~b}_{1}+b_{2}+b_{3}\right)$ if $R(a)<0$

\subsection{Linguistic Variable}

In more specific terms, a linguistic variable is characterized by a quintuple $(v, T(v), U, G, M)$ in which $v$ is the name of the variable; $\mathrm{T}(v)$ the term-set of $v$, that is, the collection of its linguistic values; $\mathrm{U}$ is an universe of discourse; $\mathrm{G}$ is a syntactic rule which generates the terms in $\mathrm{T}(\mathrm{v})$ and $\mathrm{M}$ is a semantic rule which associates with each linguistic value $X$ its meaning, $M(X)$, where $M(X)$ denotes a fuzzy subset of $U$. The meaning of a linguistic value $\mathrm{X}$ is characterized by a compatibility function, $\boldsymbol{C}: U \rightarrow[0, \mathrm{I}]$, which associates with each u in $\mathrm{U}$ its compatibility with $\mathrm{X}$

\section{Assignment Method}

The assignment problem can be stated in the form of $\mathrm{n} \mathrm{x} \mathrm{n} \mathrm{cost} \mathrm{matrix}\left[\mathrm{c}_{\mathrm{ij}}\right]$ of real numbers as given in the following table

\begin{tabular}{|c|c|c|c|c|c|}
\hline $\begin{array}{r}\text { Jobs } \rightarrow \\
\text { Persons } \\
\downarrow\end{array}$ & 1 & 2 & 3 & ---j--- & $\mathrm{n}$ \\
\hline 1 & $\mathrm{C}_{11}$ & $\mathrm{C}_{12}$ & $\mathrm{C}_{13}$ & $--C_{1 j^{--}}$ & $\mathrm{C}_{1 \mathrm{n}}$ \\
\hline 2 & $\mathrm{C}_{21}$ & $\mathrm{C}_{22}$ & $\mathrm{C}_{23}$ & $-\mathrm{C}_{2 \mathrm{j}^{--}}$ & $\mathrm{C}_{2 \mathrm{n}}$ \\
\hline- & - & - & - & - & - \\
\hline- & - & - & - & - & - \\
\hline I & $\mathrm{C}_{\mathrm{i} 1}$ & $\mathrm{C}_{\mathrm{i} 2}$ & $\mathrm{C}_{\mathrm{i} 3}$ & $--C_{i j^{\prime}-}$ & $\mathrm{C}_{\text {in }}$ \\
\hline - & & & & & \\
\hline $\mathrm{N}$ & $\mathrm{C}_{\mathrm{n} 1}$ & $\mathrm{C}_{\mathrm{n} 2}$ & $\mathrm{C}_{\mathrm{n} 3}$ & $--C_{n j^{--}}$ & $\mathrm{C}_{\mathrm{nn}}$ \\
\hline
\end{tabular}

Mathematically assignment problem can be stated as

Minimize $z=\sum_{i=1}^{n} \sum_{j=1}^{n} c_{i j} \quad x_{i j}$ where $\mathrm{i}=1,2,3 \ldots . \mathrm{n}, \mathrm{j}=1,2,3 \ldots \mathrm{n}$

Subject to

$\sum_{i=1}^{n} x_{i j}=1, \quad \mathrm{i}=1,2,3 \ldots \mathrm{n}$

$\sum_{j=1}^{n} x_{i j}=1 \quad \mathrm{j}=1,2,3 \ldots . \mathrm{n} \quad x_{i j} \in\{0,1\}$

where $x_{i j}=\left\{\begin{array}{c}1 \text { if the } i^{\text {th }} \text { person is assigned the } j^{\text {th }} \text { job } \\ 0\end{array}\right.$

is the decision variable denoting the assignment of the person $\mathrm{i}$ to job $\mathrm{j}$, Cij is the cost of assigning the $\mathrm{j}^{\text {th }}$ job to the $\mathrm{i}^{\text {th }}$ person.

The objective is to minimize the total cost of assigning all the jobs to the available persons. (One job to one person). When the costs $\tilde{c}_{i j}$ are fuzzy numbers, then the fuzzy assignment problem becomes 
$Y(\tilde{z})=\sum_{i=1}^{n} \sum_{j=1}^{n} Y\left(\tilde{c}_{i j}\right) \quad x_{i j}$

Subject to the same conditions (1)

\subsection{Algorithm for the proposed method : ( Balanced/Unbalanced assignment problem )}

If the number of rows is not equal to the number of columns then the problem is termed as unbalanced assignment problem then this problem into change balanced assignment problem as follows necessary number of dummy row (s) / column(s) are added such that the cost matrix is a square matrix the values for the entries in the dummy row (s) / column(s) are assumed to be zero.

By defuzzifying the fuzzy cost coefficients into crisp coefficients by centriod ranking formula we can solve. This problem is obviously the crisp assignment problem of the form (1) which can be solved by Ones Assignment Method.

step 1.

In a minimization (maximization) case, find the minimum (maximum) element of each row in the assignment matrix (say $a i$ ) and write it on the right hand side of the matrix. Then divide each element of ith row of the matrix by ai. These operations create at least one ones in each rows.

In term of ones for each row and column do assignment, otherwise go to step 2.

step 2.

Find the minimum (maximum) element of each column in assignment matrix (say $b j$ ), and write it below jth column. Then divide each element of jth column of the matrix by $b j$.These operations create at least one ones in each columns. Make assignment in terms of ones.If no feasible assignment can be achieved from step (1) and (2) then go to step3.

Note: In a maximization case, the end of step 2 we have a fuzzy matrix, which all elements are belong to [0, 1], and the greatest element is one [4].

step 3.

Draw the minimum number of lines to cover all the ones of the matrix. If the number of drawn lines less than $n$, then the complete assignment is not possible, while if the number of lines is exactly equal to $n$, then the complete assignment is obtained

step 4.

If a complete assignment program is not possible in step 3 , then select the smallest

( largest) element (say dij) out of those which do not lie on any of the lines in the above matrix. Then divide by dij each element of the uncovered rows or columns, which dij lies on it. This operation create some new ones to this row or column.

If still a complete optimal assignment is not achieved in this new matrix, then use step 4 and 3 iteratively. By repeating the same procedure the optimal assignment will be obtained.

Priority, plays an important role in this method, When we want to assign

the ones.

\section{Priority rule.}

For maximization (minimization) assignment problem, assign the ones on the rows which have greatest (smallest) element on the right hand side, respectively.

\section{Numerical example}

Consider the following fuzzy assignment problem. Assign the four jobs to the four machines so as to minimize the total cost.

$W$
$X$
$Y$
$Z$$\left[\begin{array}{cccc}(9,10,11) & (4,5,6) & (12,13,14) & (14,15,16) \\ (9,10,11) & (8,9,10) & (17,18,19) & (2,3,4) \\ (4,5,6) & (10,7,8) & ((2,3,4) & (1,2,3) \\ & (8,112) & (8,9,10) & (6,7,8)\end{array}\right]$

Defuzzyifying using the given ranking method. We have

1
$W$
$X$
$Y$$\left[\begin{array}{cccc}10 & 5 & 3 & 4 \\ 3 & 9 & 18 & 15 \\ 10 & 7 & 3 & 2 \\ 5 & 11 & 9 & 7\end{array}\right]$

Find the minimum (maximum) element of each row in the assignment matrix (say ai) and write it on the right hand side of the matrix. Then divide each element of ith row of the matrix by ai. 


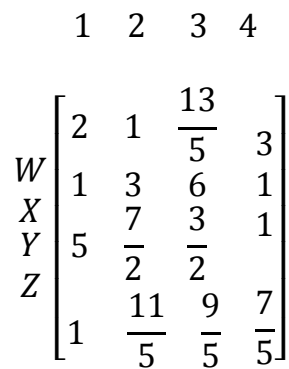

Find the minimum( maximum) element of each column in assignment matrix (say $b j$ ), and write it below jth column

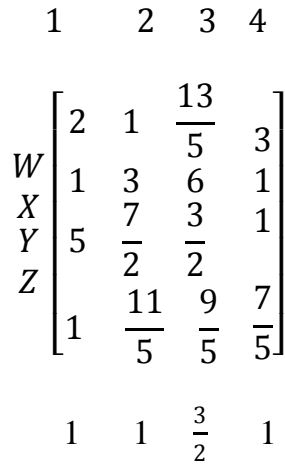

Then divide each element of jth column of the matrix by $b j$.These operations create at least one ones in each columns. Make assignment in terms of ones.

\begin{tabular}{|c|c|c|c|c|}
\hline & 1 & 2 & 3 & 4 \\
\hline & 2 & (1) & 26 & 3 \\
\hline$W$ & & & 12 & \\
\hline & 1 & 3 & $\overline{3}$ & (1) \\
\hline & & $\frac{7}{-}$ & (1) & \\
\hline$Z$ & $\begin{array}{c}5 \\
(1)\end{array}$ & $\begin{array}{c}\overline{2} \\
11\end{array}$ & 18 & 7 \\
\hline & & $\overline{15}$ & $\overline{15}$ & $\overline{5}$ \\
\hline
\end{tabular}

The optimal assignment schedule is $W \rightarrow 2, X \rightarrow 4, Y \rightarrow 3, Z \rightarrow 1$, the minimum cost is 16 .

\section{Conclusions}

In this paper, the assignment costs are considered as linguistic variables represented by fuzzy numbers. Thus the fuzzy assignment problem has been transformed into crisp balanced and unbalanced assignment problem using ranking method. Here we have shown that the fuzzy assignment problems of qualitative nature can be solved in an effective way by ones assignment method. This technique can also be tried in solving the problems like balanced and unbalanced Transportation problems, Transshipment, project scheduling problems, network flow problems etc. This research work is under process for hexagonal and octagonal fuzzy numbers.

\section{References}

[1]. $\quad$ Adamo J. M., "Fuzzy decision trees," Fuzzy Sets and Systems, vol. 4, no. 3, pp. 207-219, 1980

[2]. Baas and H. K. wakernaak, "Rating and ranking of multiple-aspect alternatives using fuzzy sets," Automatica, vol. 13, no. 1, pp. 47-58, 1977.

[3]. Baldwin J. F. and Guild N. C. F., "Comparison of fuzzy sets on the same decision space," Fuzzy Sets and Systems, vol. 2, no. 3, pp. 213-231, 1979.

[4]. Bortolan .G. and Degani, R. "A review of some methods for ranking fuzzy subsets," Fuzzy Sets and Systems, vol. 15, no. 1,pp. 1$19,1985$.

[5]. Chang W., "Ranking of fuzzy utilities with triangular membership functions," Proceedings of International Conference on Policy Analysis and Systems, pp. 263-272, 1981.

[6]. Chen S.H, "Ranking fuzzy numbers with maximizing set and minimizing set," Fuzzy Sets and Systems, vol. 17, no. 2, pp. 113-129, 1985. 
[7]. Chen, S.J., Chen, S.M.: "Fuzzy Risk Analysis based on the Ranking of Generalized Trapezoidal Fuzzy Numbers". Applied Intelligence 26, 1-11 (2007)

[8]. Delgado M., Verdegay J. L., and Vila M. A., “A procedure for ranking fuzzy numbers using fuzzy relations,” Fuzzy Sets and Systems, vol. 26, no. 1, pp. 49-62, 1988.

[9]. Dubois D. and Prade, H. "Ranking fuzzy numbers in the setting of possibility theory," Information Sciences, vol. 30, no.3, pp. 183-224, 1983.

[10]. Dubois.D and H. Prade,H, "The mean value of a fuzzy number," Fuzzy Sets and Systems, vol. 24, no. 3, pp. 279-300, 1987.

[11]. Fateen Najwa Azman* and Lazim Abdullah "Ranking Fuzzy Numbers by Centroid Method" Malaysian Journal of Fundamental \& Applied Sciences, vol.8, no.3 ,pp. 121-125,2012,

[12]. Hadi Basirzadeh," Ones Assignment Method for Solving Assignment Problems", Applied Mathematical Sciences, Vol. 6, 2012, no. 47, $2345-2355$,

[13]. Jain. R., “A procedure for multi aspect decision making using fuzzy sets,” International Journal of Systems Science, vol. 8, no.1, pp. $1-7,1978$.

[14]. Jain.R. "Decision making in the presence of fuzzy variables,"IEEE Transactions on Systems, Man and Cybernetics, vol. 6, no.10, pp. 698-703, 1976.

[15]. Kerre E., "The use of fuzzy set theory in electro cardiological diagnostics," in Approximate Reasoning in Decision-Analysis, M. M. Gupta and E. Sanchez, Eds., pp. 277-282, North Holland Publishing, Amsterdam, The Netherlands, 1982.

[16]. Lee.E.S and Li.R.J, "Comparison of fuzzy numbers based on the probability measure of fuzzy events," Computers and Mathematics with Applications, vol. 15, no. 10, pp. 887-896, 1988.

[17]. Murakami.S, Maeda.H, and Imamura.S,"Fuzzy decision analysis on the development of centralized regional energy control system," in Proceedings of the IFAC on Fuzzy Information, Knowledge Representation and Decision Analysis, pp. 353-358, Marseille, France, 1983

[18]. Yager R. R., “A procedure for ordering fuzzy subsets of the unit interval,” Information Sciences, vol. 24, no. 2, pp. 143-161,1981.

[19]. Yager R. R., "On choosing between fuzzy subsets," Kybernetes,vol. 9, no. 2, pp. 151-154, 1980.

[20]. Yager.R.R, "Ranking fuzzy subsets over the unit interval," in Proceedings of the IEEE Conference on Decision and Control (CDC '78), pp. 1435-1437, Albuquerque, NM, USA, August 1978.

[21]. Zadeh, L. A "Fuzzy sets," Information and Control, vol. 8, no.3, pp. 338-353, 1965.

[22]. Zadeh, L. A., "The concept of a linguistic variable and its application to approximate reasoning", Part 1, 2 and 3,Information Sciences, Vol.8, pp.199- 249, 1975; Vol.9, pp.43-58, 1976 